\title{
Recent updates of therapeutic strategy of esophagogastric junction adenocarcinoma
}

\author{
Suguru Maruyama, Yu Imamura, Yasukazu Kanie, Kei Sakamoto, Daisuke Fujiwara, Akihiko Okamura, Jun \\ Kanamori, Masayuki Watanabe
}

Department of Gastroenterological Surgery, Cancer Institute Hospital of Japanese Foundation of Cancer Research, Tokyo 1358550, Japan.

Correspondence to: Dr. Yu Imamura, Department of Gastroenterological Surgery, Cancer Institute Hospital of Japanese Foundation of Cancer Research, 3-8-31, Ariake, Koto, Tokyo 135-8550, Japan. E-mail: yu.imamura@jfcr.or.jp

How to cite this article: Maruyama S, Imamura Y, Kanie Y, Sakamoto K, Fujiwara D, Okamura A, Kanamori J, Watanabe M. Recent updates of therapeutic strategy of esophagogastric junction adenocarcinoma. J Cancer Metastasis Treat 2021;7:53. https://dx.doi.org/10.20517/2394-4722.2021.113

Received: 8 May 2021 First Decision: 29 Jun 2021 Revised: 11 Jul 2021 Accepted: 23 Jul 2021 First online: 3 Aug 2021

Academic Editors: Lucio Miele, William P. Schiemann Copy Editor: Yue-Yue Zhang Production Editor: Yue-Yue Zhang

\begin{abstract}
The incidence of esophagogastric junction (EGJ) adenocarcinoma has been increasing in Asian countries. Despite the recent advances in multidisciplinary treatments, EGJ adenocarcinoma remains aggressive with unfavorable outcomes. Regarding surgical strategy, EGJ adenocarcinoma arises between the esophagus and the stomach, and thus tumor cells spread through the lymphatic system both upward to the mediastinum and downward to the abdomen. Nevertheless, an optimal extent of lymphadenectomy remains controversial. Regarding drug therapy, the latest topic in gastric and EGJ adenocarcinoma is trastuzumab deruxtecan, which is an antibody-drug conjugate consisting of an anti-HER2 antibody. In addition, many clinical trials have recently demonstrated the efficacy of immune checkpoint inhibitors. Meanwhile, recent advances in sequencing technology have revealed that gastroesophageal adenocarcinoma could be categorized into four molecular subtypes: epstein-Barr virusassociated, high-level microsatellite instability, genomically stable, and chromosomal instability tumors. Furthermore, these subtypes show distinct clinical phenotypes and molecular alterations. We review the current surgical strategy and drug treatment such as molecular-targeted agents, immune checkpoint inhibitors, and molecular-subtype-based therapeutic strategies in EGJ adenocarcinoma. Clinical and molecular characteristics and response to immune checkpoint inhibitors differ among molecular subtypes. Treatment strategies based on molecular subtypes may be clinically beneficial for patients with EGJ adenocarcinoma.
\end{abstract}


Keywords: Esophagogastric junction adenocarcinoma, Barrett's esophagus, microsatellite instability, molecular subtype

\section{INTRODUCTION}

In western countries, the incidence of adenocarcinoma of the esophagogastric junction (EGJ) has dramatically increased in the last few decades ${ }^{[1-3]}$. In Asia, the prevalence of this tumor seems to be rising, as Helicobacter pylori infections become less $\operatorname{common}^{[4,5]}$. Despite the recent improvements in next generation sequencing techniques and molecular targeting treatments, EGJ adenocarcinoma remains an aggressive malignant disease with unfavorable outcomes. Regarding surgical management of this tumor, an optimal extent of lymphadenectomy is still controversial. EGJ adenocarcinoma including Barrett's adenocarcinoma, and adenocarcinoma of the gastric cardia with esophageal invasion ${ }^{[6]}$ shares molecular characteristics with gastric adenocarcinoma ${ }^{[7,8]}$, whereas tumor cells can spread more widely than gastric cancer due to the bidirectional lymphatic drainage routes (mediastinal and abdominal $)^{[9,10]}$. To improve the therapeutic strategy for this tumor, we review the previous studies investigating recent surgical management, particularly in lymph node dissection, and drug treatment based on molecular-targeted agents, immune checkpoint inhibitors and molecular subtype-based therapeutic strategies.

\section{SURGICAL TREATMENT (LYMPH NODE DISSECTION)}

EGJ adenocarcinoma dominates across the thorax and abdomen with various extents. In clinical practice, the Siewert classification is widely used for specifying the tumor location of EGJ adenocarcinoma as follows: Type I, the epicenter of tumor is located between 1 and $5 \mathrm{~cm}$ proximal to the anatomical EGJ; Type II, the epicenter is located between $1 \mathrm{~cm}$ proximal to and $2 \mathrm{~cm}$ distal from the EGJ; and Type III, a gastric tumor with esophageal invasion in which the tumor epicenter is located between 2 and $5 \mathrm{~cm}$ distal from the $\mathrm{EG}^{[11]}$. Considering that Barrett's esophagus is a replacement of normal squamous mucosae with columnar epithelium under gastroesophageal reflux disease, Barrett's adenocarcinoma is presumably located on the proximal side of the EGJ (Siewert Type I) compared to cardiac tumors ${ }^{[12]}$.

Siewert classification is useful when surgeons select a surgical approach. Siewert Type I tumors usually need a transthoracic approach with dissection of mediastinal nodes. In contrast, Siewert Type II-III tumors can be resected by transhiatal approach alone when technically possible. Thus far, several studies have examined the clinicopathological and prognostic features by comparative analysis among Siewert Type I-III tumors $^{[13-19]}$. The surgical outcome is still conflicting; some studies suggested Siewert Type III has the worst outcome, but others observed no significant differences among the Siewert type ${ }^{[13,16,18]}$. Nodal metastases ${ }^{[13]}$, Ro resection ${ }^{[14]}$, and lymphovascular invasion ${ }^{[17]}$ appeared to be prognostic factors of EGJ adenocarcinoma. Our retrospective multicenter cohort study revealed that Siewert Type I patients with Stage II-III tumors had significantly unfavorable outcomes. Over half of such patients experienced lymph node recurrence ${ }^{[20]}$. In locally advanced cases such as Stage II-III diseases, Siewert Type I tumors can spread widely through the lymphatics, leading to multi-field lymph node metastases. A hiatal hernia may concern the pattern of lymphatic spread of tumor cells in EGJ adenocarcinoma. We previously reported frequent mediastinal lymph node recurrences in EGJ adenocarcinoma cases accompanied by hiatal hernia, compared to those without hiatal hernia ${ }^{[21]}$.

The Japan Esophageal Society (JES) and Japanese Gastric Cancer Association (JGCA) defined the lymph node stations for esophageal and stomach cancers, respectively ${ }^{[22,23]}$. Thus far, two studies have investigated the lymph node metastasis rate according to the lymph node stations, as a collaborative study between JES and JGCA. The first study was a multicenter retrospective study collecting EGJ tumors determined by Nishi 
classification, in which a tumor center is located between $2 \mathrm{~cm}$ proximal to and $2 \mathrm{~cm}$ distal from EGJ. Among 2807 cases, limiting the cases to less than $40 \mathrm{~mm}$ in diameter ${ }^{[22]}$, there were 2384 (84.9\%) cases with adenocarcinoma, 370 (13.2\%) with squamous cell carcinoma (SCC), and $53(1.9 \%)$ with other histological types of malignancy. Based on their observations, lower mediastinal node dissection (No. 110) might improve survival in the patients with esophagus-predominant tumors. However, they could show no conclusive result for the efficacy of middle or upper mediastinal node dissection due to the scarcity of dissected cases.

Another prospective nationwide multicenter study reported the mapping of lymph node metastasis of Nishi-defined EGJ tumors, including 332 cases of adenocarcinoma $(91.5 \%)$ and 31 with SCC $(8.5 \%)^{[23]}$. They classified regional nodes into the following three categories: Category 1 as the nodes with metastasis rate of $>10 \%$, Category 2 as $5 \%-10 \%$, and Category 3 as $<5 \%$. They suggested a strong recommendation of lymph node dissection for Category 1 and a weak recommendation for Category 2, but no recommendation for Category 3. They indicated that the lower thoracic para-esophageal node (No. 110) should be Category 1 for tumors with esophageal invasion of more than $2.0 \mathrm{~cm}$. The supradiaphragmatic node (No. 111) should be Category 1, while the posterior mediastinal node (No. 112) should be Category 2 for tumors with the esophageal invasion of more than $4.0 \mathrm{~cm}$. Among the middle mediastinal nodes, the subcarinal node (No. 107), the middle thoracic para-esophageal node (No. 108), and the left main bronchus node (No. 109L) were included in Category 2 for tumors with the esophageal invasion of more than $4.0 \mathrm{~cm}$. In the upper mediastinum, the authors recommended dissection of the right recurrent laryngeal nerve node (106recR) as Category 1 for tumors with the esophageal invasion of more than $4.0 \mathrm{~cm}$. In contrast, the left recurrent laryngeal nerve node (No. 106recL) was classified into Category 3, even in cases with significant esophageal invasion. As the recommendation is thus far based only on the metastatic rates, we need to wait for the survival outcomes for better clinical recommendations.

Regarding the surgical approach for lower mediastinal node dissection, the transhiatal approach is known to have a lower risk of pneumonia than the left thoracoabdominal approach in patients with Siewert Type IIIII tumors with less than $3 \mathrm{~cm}$ esophageal invasion, referring to the results of JCOG 9502, a Phase III trial ${ }^{[24]}$. Meanwhile, there is still a lack of evidence for dissection of upper to middle thoracic nodes and the appropriate surgical approaches.

\section{DRUG THERAPY}

\section{Molecular-targeted agents}

Molecular-targeted drugs have been created for various types of cancers. We summarize the previously investigated clinical trials of molecular targeting agents for EGJ and gastric adenocarcinoma [Table 1].

Trastuzumab is a monoclonal antibody targeting human epidermal growth factor receptor 2 (HER2). The ToGA trial (Phase III trial, including 106 cases with EGJ adenocarcinoma and 478 cases with gastric adenocarcinoma) assessed the safety and survival benefit of trastuzumab plus first-line chemotherapy (5fluorouracil and platinum or capecitabine and platinum) for advanced tumors with HER2-amplified esophagogastric adenocarcinoma in 2010. HER2 amplification or overexpression was more prevalent in EGJ cancer $(33.2 \%)$ compared to that in gastric cancer $(20.9 \%)(P<0.001)$. The median overall survival (OS) was significantly better in the trastuzumab plus chemotherapy group than that in the chemotherapy alone group [median 13.8 months; 95\% confidence interval (CI): 12-16 months vs. median 11.1 months; 95\%CI: 10-13 months] [hazard ratio (HR) $=0.74 ; 95 \% \mathrm{CI}$ : 0.60-0.91; $P=0.0046]^{[25]}$. The National Comprehensive Cancer Network guideline recommends combination use of trastuzumab with any chemotherapeutic agents in patients with HER2-amplified or -overexpressing EGJ adenocarcinoma as well as gastric tumors. 
Table 1. Clinical trials testing targeted therapies for EGJ and gastric adenocarcinoma

\begin{tabular}{llllll}
\hline Target & Trial & Patients (EGJ) & Treatment & Outcome & Primary endpoint \\
\hline HER2 & ToGA & $594(106)$ & XP vs. XP + trastuzumab & Positive & OS \\
HER2 & LOGiC & $545(49)$ & CapeOx vs. CapeOx + lapatinib & Negative & OS \\
EGFR & EXPAND & $904(144)$ & XPvs. XP + cetuximab & Negative & OS \\
EGFR & REAL3 & $553(169)$ & EOC vs. EOC + panitumumab & Negative & OS \\
MET & RILOMET-1 & $609(124)$ & ECX vs. ECX + rilotumumab & Negative & OS \\
MET/HGF & METGastric & $562(130)$ & mFOLFOX6 vs. mFOLFOX6 + onartuzumab & Negative & OS \\
VEGFR-A & AVAGAST & $774(130)$ & XPvs. XP + bevacizumab & Negative & OS \\
VEGFR2 & RAINBOW & $665(137)$ & Paclitaxel vs. Paclitaxel + ramucirumab & Positive & OS \\
VEGFR2 & REGARD & $355(90)$ & Placebo vs. ramucirumab & Positive & OS \\
HER2 & TyTAN & $261(0)$ & Paclitaxel or docetaxel vs. Trastuzumab-emtansine & Negative & OS \\
HER2 & GATSBY & $345(110)$ & Paclitaxel vs. Paclitaxel + lapatinib & Negative & OS \\
mTOR & GRANITE-1 & $656(187)$ & Placebo vs. Everolimus & Negative & OS \\
\hline
\end{tabular}

EGJ: Esophagogastric junction; HER2: human epidermal growth factor receptor 2; XP: capecitabine plus cisplatin; CapeOx: capecitabine plus oxaliplatin; EGFR: epidermal growth factor receptor; EOC: epirubicin, oxaliplatin and capecitabine; ECX: epirubicin, cisplatin and capecitabine; HGF: hepatocyte growth factor; mFOLFOX: levofolinate, fluorouracil and oxaliplatin; VEGFR: vascular endothelial growth factor receptor; mTOR: mammalian target of rapamycin.

Ramucirumab is a human immunoglobulin (Ig) G1 monoclonal antibody that antagonizes vascular endothelial growth factor receptor-2. Two clinical trials have shown a survival advantage of ramucirumab, as monotherapy or combined with paclitaxel, in the second or more regiment setting for advanced EGJ adenocarcinoma. The REGARD and RAINBOW trials have successfully demonstrated the survival benefits of ramucirumab in the second-line regimen for advanced unresectable esophagogastric adenocarcinomas ${ }^{[2,2,2]}$. Hence, new investigational strategies adding ramucirumab may be intriguing for resectable EGJ adenocarcinoma using effective drugs for advanced setting.

Bevacizumab, a monoclonal medicine targeting vascular endothelial growth factor A, inhibits tumor progression in preclinical settings. Unfortunately, the AVAGAST trial did not show any survival benefit of bevacizumab $^{[28-30]}$. Lapatinib is the dual inhibitor of epidermal growth factor receptor (EGFR) and HER2. Lapatinib showed no additional survival benefit to the combination therapy of capecitabine plus oxaliplatin in the first-line setting of HER2-amplified/overexpressing esophagogastric adenocarcinoma (the TRIO$013 /$ LOGiC trial ${ }^{[31]}$. Cetuximab, an EGFR antibody, is widely used for the patients with advanced diseases of head and neck, non-small-cell lung, and KRAS wild-type colorectal cancers ${ }^{[32-35]}$. The EXPAND trial has failed in demonstrating a survival benefit in the first-line use of cetuximab in addition to capecitabine plus cisplatin $^{[36]}$. Panitumumab, which is another monoclonal antibody targeting EGFR, significantly improved progression-free survival in patients with advanced colorectal cancer ${ }^{[37]}$. However, no survival benefit was observed in adding panitumumab to a triplet regimen using epirubicin, oxaliplatin, and capecitabine (REAL3 Phase III trial) ${ }^{[38]}$. Additionally, MET inhibitors, including rilotumumab and onartuzumab, and everolimus (a mammalian target of rapamycin inhibitor) conferred no survival benefits in EGJ cancer ${ }^{[39-41]}$.

As described above, many Phase III studies using molecular-targeted drugs have reported negative results. The latest topic regarding molecular-targeted agents in gastric and EGJ adenocarcinoma is the DESTINYGastric01 trial. Trastuzumab deruxtecan (DS8201) is an antibody-drug conjugate consisting of an antiHER2 antibody, a cleavable tetra-peptide-based linker, and a cytotoxic topoisomerase I inhibitor. The DESTINY-Gastrico1 trial in 2020 (Phase II trial, including 24 cases with EGJ adenocarcinoma and 163 cases with gastric adenocarcinoma) evaluated the objective response of trastuzumab deruxtecan compared with chemotherapy in patients with HER2-positive advanced gastric cancer. Patients who progressed while they 
were receiving at least two previous therapies, including trastuzumab, were randomly assigned in a 2:1 ratio to receive trastuzumab deruxtecan or physician's choice of chemotherapy (irinotecan or paclitaxel). An objective response was reported in $51 \%$ of the patients in the trastuzumab deruxtecan group, as compared with $14 \%$ of those in the physician's choice group $(P<0.001)$. OS was longer with trastuzumab deruxtecan than with chemotherapy (median, 12.5 vs. 8.4 months; $\mathrm{HR}=0.59 ; 95 \% \mathrm{CI}: 0.39-0.88 ; P=0.01)^{[42]}$.

\section{Immune checkpoint inhibitors}

Immune checkpoint inhibitors (ICIs) have emerged with remarkable anti-tumor activity against esophagogastric adenocarcinoma. Programmed death protein 1 (PD-1), PD-1 ligand 1 (PD-L1), and cytotoxic T lymphocyte protein 4 (CTLA4) are key molecules regulating the immune escape mechanism in cancer ${ }^{[43]}$. Nivolumab is a human IgG4 monoclonal antibody (mAb) against PD-1. The ATTRACTION-2 trial (Phase III) has demonstrated a significant survival prolongation of nivolumab in the third-line therapy for advanced gastric tumors ${ }^{[44]}$. In addition, the ATTRACTION-4 trial (Phase II) has demonstrated that nivolumab in combination with chemotherapy in patients with untreated unresectable advanced or recurrent gastric cancer may be a potential therapeutic option ${ }^{[45]}$. In addition, according to the recent CheckMate-649 study, nivolumab plus chemotherapy represents a new possibility for standard first-line treatment for advanced gastric, EGJ, and esophageal adenocarcinoma ${ }^{[46]}$. In addition, adjuvant administration of nivolumab has just been shown to be effective in patients with Stage II-III tumors in esophagus or EGJ cancer (CheckMate-577) ${ }^{[47]}$. Pembrolizumab is another humanized high-affinity IgG4 $\mathrm{mAb}$ acting against PD-1. The KEYNOTE-059 trial has reported a favorable overall response rate of $11.6 \%$ in patients with advanced gastric cancer treated with pembrolizumab in a Phase II setting ${ }^{[48]}$. However, the KEYNOTE-061 study, a Phase III trial comparing pembrolizumab with paclitaxel as the second-line treatment for advanced gastric or EGJ tumors, has failed to meet the primary endpoint of improving survival ${ }^{[49]}$. In addition, the KEYNOTE-062 study (Phase III) demonstrated that pembrolizumab or pembrolizumab plus chemotherapy was not superior to chemotherapy according to OS and progressionfree survival in patients with untreated advanced gastric cancer ${ }^{[50]}$. A Phase III trial of pembrolizumab plus chemotherapy vs. chemotherapy as neoadjuvant/adjuvant treatment for resectable gastric or EGJ adenocarcinoma is ongoing (KEYNOTE-585) ${ }^{[51]}$. Avelumab, another ICI targeting PD-L1, could not show survival benefits over chemotherapy as a third-line treatment for advanced gastric or EGJ cancer (JAVELIN Gastric 300) ${ }^{[52]}$. Meanwhile, in western countries, perioperative chemotherapy with fluorouracil plus leucovorin, oxaliplatin, and docetaxel (FLOT4) was selected for patients with locally advanced, resectable gastric and EGJ cancer ${ }^{[53]}$. A Phase II trial to evaluate the safety, efficacy, and toxicities of perioperative chemo-immunotherapy with avelumab and FLOT (fluorouracil plus leucovorin, oxaliplatin, and docetaxel) is ongoing (ICONIC trial). Ipilimumab is a $\mathrm{mAb}$ that activates the immune system by targeting CTLA4. In a Phase II trial, the nivolumab plus ipilimumab group showed a higher OS rate than the nivolumab alone group (Checkmate-032) ${ }^{[54]}$. However, the higher incidence of immune-related adverse events after nivolumab plus ipilimumab treatment than after nivolumab alone must be considered ${ }^{[55]}$.

\section{Molecular subtype-based therapeutic strategy}

Thus far, pathological classification has been a major type of tumor classification in gastroesophageal tumors. Recently, the next-generation sequencing technology developed molecular taxonomies in various types of malignancies, including gastroesophageal tumors, utilizing whole genome sequencing, whole exon sequencing, RNA sequencing, comprehensive methylation assay, and proteomic assays ${ }^{[8,56,57]}$. The Cancer Genome Atlas Network (TCGA) has demonstrated four molecular subtypes in gastroesophageal adenocarcinoma as follows; Epstein-Barr virus (EBV)-associated, high-level microsatellite instability (MSI$\mathrm{H})$, genomically stable (GS), and chromosomal instability (CIN) tumors ${ }^{[7,8]}$. 
EBV-associated tumors show $\mathrm{CPG}$ islandmethylator phenotype displaying $C D K N 2 A$ silencing; frequent mutations in PIK3CA, ARID1A, and BCOR; and gene amplification in ERBB2, JAK2/PD-L1/2, and $P I K 3 C A^{[7,8]}$. In addition, this subtype is suggested to be immune reactive. Although EBV-associated tumors seem to account for only a small fraction of EGJ adenocarcinomas, recent comprehensive genomic analyses have suggested their sensitivity to ICIs through PD-L1 or PD-L2 ${ }^{[7,58]}$.

MSI-H tumors, which are uncommon in EGJ, harbor hypermutation, hypermethylation, $M L H 1$ silencing, and frequent mutations in ARID1A, RNF43, PIK3CA, and KRAS and are immune reactive ${ }^{[7,8,59]}$. According to an exploratory investigation utilizing the data from the Medical Research Council Adjuvant Gastric Infusional Chemotherapy trial, a favorable outcome was associated with MSI-H subtype. However, MSI-H was a predictor of chemo-refractory tumors. In addition, MSI-H is associated with less lymph node metastasis and a favorable outcome ${ }^{[60]}$. Therefore, surgery alone may be adequate to cure operable MSI-H EGJ adenocarcinoma ${ }^{[6]]}$. Since hypermutated tumors produce neoantigens, MSI-H tumors are already known as immunogenic, and anti-tumor immunity is able to be activated against the neoantigens released into the tumor microenvironment ${ }^{[62]}$. Although nivolumab significantly conferred a survival advantage in a RCT involving patients with metastatic disease of esophagogastric adenocarcinoma, regardless of molecular subtypes, it may be more effective in EBV-associated or MSI-H tumors ${ }^{[44,58]}$.

GS tumors commonly present diffuse-type histology defined by the Lauren classification. This subtype frequently possesses mutations in $R H O A$ and $C D H 1$, and oncogenic gene fusion of CLDN18-ARHGAP26 was also frequently detected ${ }^{[7,8]}$. Besides these major alterations, BRCA1-2, CTNNA1, and RAD51C were detected in this type of gastric adenocarcinoma ${ }^{[63-66]}$. CIN tumors can be described as characterized with structural chromosomal instability, whole-genome doubling, and oncogenic gene amplification particularly in the RTK-RAS pathway and cell cycle-related genes ${ }^{[7,8,67,68]}$. We developed a novel targeted therapy focusing on KRAS-amplification in EGJ adenocarcinoma ${ }^{[69]}$. KRAS-amplified malignant cells possess a large amount of inactive KRAS-GDP. Under mitogen-activated protein kinase inhibition, KRAS-amplified tumor cells do not react due to adaptive response by mobilizing inactive KRAS-GDP to the active state of KRAS-GTP. This adaptive reaction is able to be suppressed by blocking SOS1 and SOS2, both of which are guanine-exchange factors. In addition, the protein tyrosine phosphatase SHP2 is similarly inhibited. Hence, a combination of an MEK inhibitor and SHP2 blockade may be a promising medicine for CIN-subtype in EGJ adenocarcinoma. Other molecules and immune checkpoint molecules need to be evaluated for GS or CIN tumors.

The molecular characteristics of EGJ and gastric adenocarcinoma are different. Firstly, the distribution of molecular subtypes differs across tumor locations, among EGJ (cardia), gastric (fundus/body), and gastric antrum (pylorus) ${ }^{[7,8]}$. Actually, 84.8\% of EGJ adenocarcinoma (combining Siewert Type I-III tumors) were classified into CIN subtype. In contrast, MSI-H (4.2\%) and EBV (3.6\%) subtypes were rare in EGJ adenocarcinoma in the TCGA study ${ }^{[8]}$. In particular, no MSI-H or EBV subtype was detected in Siewert Type I of esophageal adenocarcinoma. These trends were also observed in our recent report ${ }^{[60]}$. Secondly, when focusing on CIN subtype, genetic and epigenetic alterations are different in EGJ and gastric adenocarcinoma. The TCGA esophageal study clustered CIN esophagogastric adenocarcinoma into four continuous categories, $\mathrm{C} 1$ (the most hypermethylated category) to $\mathrm{C}_{4}$ (the least methylated category) ${ }^{[8]}$. The most hypermethylated $\mathrm{C} 1$ was frequently observed in $36.6 \%$, but the least methylated $\mathrm{C} 4$ was found in $4.2 \%$ in EGJ adenocarcinoma. However, in gastric adenocarcinoma, $\mathrm{C} 1$ was only $3.4 \%$ and $\mathrm{C} 4$ increased to $23.7 \%$ in fundus or body tumor. In addition, in antrum or pyloric tumor, epigenetic changes are less common compared to EGJ tumors. For instance, epigenetic silencing of CDKN2A (also known as p16) was detected in $30 \%-40 \%$ of EGJ adenocarcinoma but in only $5 \%-6 \%$ of stomach tumors ${ }^{[8]}$. Thus, DNA demethylating 
agents may be a useful therapeutic strategy of EGJ adenocarcinoma. In addition, tumor suppressor gene alterations such as SMARCA4 deletion/mutation, RUNX1 deletion, FHIT deletion, and WWOX deletion frequently occurred in EGJ adenocarcinoma, compared to gastric tumors ${ }^{[8]}$. Some oncogenic gene amplifications such as VEGFA copy number gain were also frequently observed in EGJ adenocarcinoma compared to gastric tumors. Administrating molecular targeting agents according to both molecular subtypes and tumor location may be one of the options in future clinical trials.

\section{CONCLUSION}

We review the current surgical strategy, evidence of molecular-targeted agents, and candidate therapeutic targets in EGJ adenocarcinoma. According two collaborative studies, lower mediastinal node dissection might improve survival, however there is still a lack of evidence for dissection of upper to middle thoracic nodes and the appropriate surgical approaches. Regarding molecular analyses, a recent comprehensive genomic analysis revealed four distinct molecular subtypes based on different carcinogenic steps. Interestingly, clinical and molecular characteristics and responses to ICIs differ between molecular subtypes. A molecular subtype-based treatment strategy may be clinically beneficial for patients with EGJ adenocarcinoma.

\section{DECLARATIONS}

\section{Authors' contributions}

Wrote the manuscript: Maruyama S

Designed the research and helped to draft the manuscript: Imamura $Y$

Made substantial contributions to the data analysis and interpretation: Kanie Y, Sakamoto K, Fujiwara D, Okamura A, Kanamori J

Designed the research and helped to draft the manuscript: Watanabe M

All authors have revised and approved the manuscript.

\section{Availability of data and materials}

Not applicable.

\section{Financial support and sponsorship}

None.

\section{Conflicts of interest}

All authors declared that there are no conflicts of interest.

\section{Ethical approval and consent to participate}

Not applicable.

\section{Consent for publication}

Not applicable.

\section{Copyright}

(c) The Author(s) 2021.

\section{REFERENCES}

1. Devesa SS, Blot WJ, Fraumeni JF, Jr. Changing patterns in the incidence of esophageal and gastric carcinoma in the United States. Cancer 1998;83:2049-53. PubMed

2. Bollschweiler E, Wolfgarten E, Gutschow C, Hölscher AH. Demographic variations in the rising incidence of esophageal adenocarcinoma in white males. Cancer 2001;92:549-55. DOI PubMed

3. Buas MF, Vaughan TL. Epidemiology and risk factors for gastroesophageal junction tumors: understanding the rising incidence of this 
disease. Semin Radiat Oncol 2013;23:3-9. DOI PubMed PMC

4. Blaser MJ. Disappearing microbiota: Helicobacter pylori protection against esophageal adenocarcinoma. Cancer Prev Res (Phila) 2008;1:308-11. DOI PubMed

5. Chow WH, Blaser MJ, Blot WJ, et al. An inverse relation between cagA + strains of helicobacter pylori infection and risk of esophageal and gastric cardia adenocarcinoma. Cancer Res 1998;58:588-90. PubMed

6. Yamada M, Kushima R, Oda I, et al. Different histological status of gastritis in superficial adenocarcinoma of the esophagogastric junction. Jpn J Clin Oncol 2014;44:65-71. DOI PubMed

7. Genome Atlas Research Network. Comprehensive molecular characterization of gastric adenocarcinoma. Nature 2014;513:202-9. DOI

8. Cancer Genome Atlas Research Network, Analysis Working Group: Asan University, BC Cancer Agency, et al. Integrated genomic characterization of oesophageal carcinoma. Nature 2017;541:169-75. DOI

9. Siewert J, Feith M, Werner M, Stein HJ. Adenocarcinoma of the esophagogastric junction: results of surgical therapy based on anatomical/topographic classification in 1,002 consecutive patients. Ann Surg 2000;232:353-61. DOI PubMed PMC

10. Grotenhuis BA, Wijnhoven BP, van Marion R, et al. The sentinel node concept in adenocarcinomas of the distal esophagus and gastroesophageal junction. J Thorac Cardiovasc Surg 2009;138:608-12. DOI PubMed

11. Siewert JR, Stein HJ. Classification of adenocarcinoma of the oesophagogastric junction. Br J Surg 1998;85:1457-9. DOI PubMed

12. Spechler SJ, Souza RF. Barrett's esophagus. N Engl J Med 2014;371:836-45. DOI PubMed

13. Hosokawa Y, Kinoshita T, Konishi M, et al. Clinicopathological features and prognostic factors of adenocarcinoma of the esophagogastric junction according to Siewert classification: experiences at a single institution in Japan. Ann Surg Oncol 2012;19:67783. DOI PubMed

14. Hasegawa S, Yoshikawa T, Cho H, Tsuburaya A, Kobayashi O. Is adenocarcinoma of the esophagogastric junction different between Japan and western countries? World J Surg 2009;33:95-103. DOI PubMed

15. Siewert JR, Feith M, Stein HJ. Biologic and clinical variations of adenocarcinoma at the esophago-gastric junction: relevance of a topographic-anatomic subclassification. J Surg Oncol 2005;90:139-46; discussion 146. DOI PubMed

16. Leers JM, DeMeester SR, Chan N, et al. Clinical characteristics, biologic behavior, and survival after esophagectomy are similar for adenocarcinoma of the gastroesophageal junction and the distal esophagus. J Thorac Cardiovasc Surg 2009;138:594-602; discussion 601. DOI PubMed

17. Rahden BH, Stein HJ, Feith M, Becker K, Siewert JR. Lymphatic vessel invasion as a prognostic factor in patients with primary resected adenocarcinomas of the esophagogastric junction. J Clin Oncol 2005;23:874-9. DOI PubMed

18. Parry K, Haverkamp L, Bruijnen RC, Siersema PD, Ruurda JP, van Hillegersberg R. Surgical treatment of adenocarcinomas of the gastro-esophageal junction. Ann Surg Oncol 2015;22:597-603. DOI PubMed

19. Ott K, Bader FG, Lordick F, Feith M, Bartels H, Siewert JR. Surgical factors influence the outcome after Ivor-Lewis esophagectomy with intrathoracic anastomosis for adenocarcinoma of the esophagogastric junction: a consecutive series of 240 patients at an experienced center. Ann Surg Oncol 2009;16:1017-25. DOI PubMed

20. Imamura Y, Watanabe M, Oki E, Morita M, Baba H. Esophagogastric junction adenocarcinoma shares characteristics with gastric adenocarcinoma: literature review and retrospective multicenter cohort study. Ann Gastroenterol Surg 2021;5:46-59. DOI PubMed PMC

21. Maruyama S, Kawaguchi Y, Akaike H, et al. Prognostic significance of hiatal hernia in patients with gastric cancer located within the upper-third of the stomach. World J Surg 2020;44:863-8. DOI PubMed

22. Yamashita H, Seto Y, Sano T, Makuuchi H, Ando N, Sasako M. Results of a nation-wide retrospective study of lymphadenectomy for esophagogastric junction carcinoma. Gastric Cancer 2017;20:69-83. DOI PubMed

23. Kurokawa Y, Takeuchi H, Doki Y, et al. Mapping of lymph node metastasis from esophagogastric junction tumors: a prospective nationwide multicenter study. Ann Surg 2021;274:120-7. DOI PubMed

24. Sasako M, Sano T, Yamamoto S, et al. Left thoracoabdominal approach versus abdominal-transhiatal approach for gastric cancer of the cardia or subcardia: a randomised controlled trial. Lancet Oncol 2006;7:644-51. DOI PubMed

25. Bang Y, Van Cutsem E, Feyereislova A, et al. Trastuzumab in combination with chemotherapy versus chemotherapy alone for treatment of HER2-positive advanced gastric or gastro-oesophageal junction cancer (ToGA): a phase 3, open-label, randomised controlled trial. Lancet 2010;376:687-97. DOI PubMed

26. Fuchs CS, Tomasek J, Yong CJ, et al. Ramucirumab monotherapy for previously treated advanced gastric or gastro-oesophageal junction adenocarcinoma (REGARD): an international, randomised, multicentre, placebo-controlled, phase 3 trial. Lancet 2014;383:31-9. DOI PubMed

27. Wilke H, Muro K, Van Cutsem E, et al. Ramucirumab plus paclitaxel versus placebo plus paclitaxel in patients with previously treated advanced gastric or gastro-oesophageal junction adenocarcinoma (RAINBOW): a double-blind, randomised phase 3 trial. Lancet Oncol 2014;15:1224-35. DOI PubMed

28. Cunningham D, Stenning SP, Smyth EC, et al. Peri-operative chemotherapy with or without bevacizumab in operable oesophagogastric adenocarcinoma (UK Medical Research Council ST03): primary analysis results of a multicentre, open-label, randomised phase 2-3 trial. Lancet Oncol 2017;18:357-70. DOI PubMed PMC

29. Gerber HP, Ferrara N. Pharmacology and pharmacodynamics of bevacizumab as monotherapy or in combination with cytotoxic therapy in preclinical studies. Cancer Res 2005;65:671-80. PubMed

30. Ohtsu A, Shah MA, Van Cutsem E, et al. Bevacizumab in combination with chemotherapy as first-line therapy in advanced gastric cancer: a randomized, double-blind, placebo-controlled phase III study. J Clin Oncol 2011;29:3968-76. DOI PubMed 
31. Hecht JR, Bang YJ, Qin SK, et al. Lapatinib in combination with capecitabine plus oxaliplatin in human epidermal growth factor receptor 2-positive advanced or metastatic gastric, esophageal, or gastroesophageal adenocarcinoma: TRIO-013/LOGiC - a randomized Phase III trial. J Clin Oncol 2016;34:443-51. DOI PubMed

32. Bokemeyer C, Bondarenko I, Hartmann JT, et al. Efficacy according to biomarker status of cetuximab plus FOLFOX-4 as first-line treatment for metastatic colorectal cancer: the OPUS study. Ann Oncol 2011;22:1535-46. DOI PubMed

33. Van Cutsem E, Köhne CH, Láng I, et al. Cetuximab plus irinotecan, fluorouracil, and leucovorin as first-line treatment for metastatic colorectal cancer: updated analysis of overall survival according to tumor KRAS and BRAF mutation status. J Clin Oncol 2011;29:2011-9. DOI PubMed

34. Vermorken JB, Mesia R, Rivera F, et al. Platinum-based chemotherapy plus cetuximab in head and neck cancer. $N$ Engl $J$ Med 2008;359:1116-27. DOI PubMed

35. Pirker R, Pereira JR, Szczesna A, et al. Cetuximab plus chemotherapy in patients with advanced non-small-cell lung cancer (FLEX): an open-label randomised phase III trial. Lancet 2009;373:1525-31. DOI PubMed

36. Lordick F, Kang Y, Chung H, et al. Capecitabine and cisplatin with or without cetuximab for patients with previously untreated advanced gastric cancer (EXPAND): a randomised, open-label phase 3 trial. Lancet Oncol 2013;14:490-9. DOI PubMed

37. Douillard JY, Siena S, Cassidy J, et al. Randomized, phase III trial of panitumumab with infusional fluorouracil, leucovorin, and oxaliplatin (FOLFOX4) versus FOLFOX4 alone as first-line treatment in patients with previously untreated metastatic colorectal cancer: the PRIME study. J Clin Oncol 2010;28:4697-705. DOI PubMed

38. Waddell T, Chau I, Cunningham D, et al. Epirubicin, oxaliplatin, and capecitabine with or without panitumumab for patients with previously untreated advanced oesophagogastric cancer (REAL3): a randomised, open-label phase 3 trial. Lancet Oncology 2013;14:481-9. DOI PubMed PMC

39. Catenacci DVT, Tebbutt NC, Davidenko I, et al. Rilotumumab plus epirubicin, cisplatin, and capecitabine as first-line therapy in advanced MET-positive gastric or gastro-oesophageal junction cancer (RILOMET-1): a randomised, double-blind, placebo-controlled, phase 3 trial. Lancet Oncol 2017;18:1467-82. DOI PubMed PMC

40. Shah MA, Bang YJ, Lordick F, et al. Effect of fluorouracil, leucovorin, and oxaliplatin with or without onartuzumab in HER2negative, MET-positive gastroesophageal adenocarcinoma: the METGastric randomized clinical trial. JAMA Oncol 2017;3:620-7. DOI PubMed PMC

41. Ohtsu A, Ajani JA, Bai YX, et al. Everolimus for previously treated advanced gastric cancer: results of the randomized, double-blind, phase III GRANITE-1 study. J Clin Oncol 2013;31:3935-43. DOI PubMed PMC

42. Shitara K, Bang YJ, Iwasa S, et al. Trastuzumab deruxtecan in previously treated HER2-positive gastric cancer. $N$ Engl J Med 2020;382:2419-30. DOI PubMed

43. Kono K, Nakajima S, Mimura K. Current status of immune checkpoint inhibitors for gastric cancer. Gastric Cancer 2020;23:565-78. DOI PubMed

44. Kang Y, Boku N, Satoh T, et al. Nivolumab in patients with advanced gastric or gastro-oesophageal junction cancer refractory to, or intolerant of, at least two previous chemotherapy regimens (ONO-4538-12, ATTRACTION-2): a randomised, double-blind, placebocontrolled, phase 3 trial. Lancet 2017;390:2461-71. DOI PubMed

45. Boku N, Ryu MH, Kato K, et al. Safety and efficacy of nivolumab in combination with S-1/capecitabine plus oxaliplatin in patients with previously untreated, unresectable, advanced, or recurrent gastric/gastroesophageal junction cancer: interim results of a randomized, phase II trial (ATTRACTION-4). Ann Oncol 2019;30:250-8. DOI PubMed PMC

46. Janjigian YY, Shitara K, Moehler M, et al. First-line nivolumab plus chemotherapy versus chemotherapy alone for advanced gastric, gastro-oesophageal junction, and oesophageal adenocarcinoma (CheckMate 649): a randomised, open-label, phase 3 trial. Lancet 2021;398:27-40. DOI PubMed

47. Kelly RJ, Ajani JA, Kuzdzal J, et al. Adjuvant nivolumab in resected esophageal or gastroesophageal junction cancer. $N$ Engl J Med 2021;384:1191-203. DOI PubMed

48. Fashoyin-Aje L, Donoghue M, Chen H, et al. FDA approval summary: pembrolizumab for recurrent locally advanced or metastatic gastric or gastroesophageal junction adenocarcinoma expressing PD-L1. Oncologist 2019;24:103-9. DOI PubMed PMC

49. Shitara K, Özgüroğlu M, Bang Y, et al. Pembrolizumab versus paclitaxel for previously treated, advanced gastric or gastrooesophageal junction cancer (KEYNOTE-061): a randomised, open-label, controlled, phase 3 trial. Lancet 2018;392:123-33. DOI PubMed

50. Shitara K, Van Cutsem E, Bang YJ, et al. Efficacy and safety of pembrolizumab or pembrolizumab plus chemotherapy vs. chemotherapy alone for patients with first-line, advanced gastric cancer: The KEYNOTE-062 Phase 3 randomized clinical trial. JAMA Oncol 2020;6:1571-80. PubMed PMC

51. Bang YJ, Van Cutsem E, Fuchs CS, et al. KEYNOTE-585: Phase III study of perioperative chemotherapy with or without pembrolizumab for gastric cancer. Future Oncol 2019;15:943-52. DOI PubMed

52. Bang YJ, Ruiz EY, Van Cutsem E, et al. Phase III, randomised trial of avelumab versus physician's choice of chemotherapy as thirdline treatment of patients with advanced gastric or gastro-oesophageal junction cancer: primary analysis of JAVELIN Gastric 300. Ann Oncol 2018;29:2052-60. DOI PubMed PMC

53. Al-batran S, Homann N, Pauligk C, et al. Perioperative chemotherapy with fluorouracil plus leucovorin, oxaliplatin, and docetaxel versus fluorouracil or capecitabine plus cisplatin and epirubicin for locally advanced, resectable gastric or gastro-oesophageal junction adenocarcinoma (FLOT4): a randomised, phase 2/3 trial. Lancet 2019;393:1948-57. DOI PubMed

54. Janjigian YY, Bendell J, Calvo E, et al. CheckMate-032 study: efficacy and safety of nivolumab and nivolumab plus ipilimumab in patients with metastatic esophagogastric cancer. J Clin Oncol 2018;36:2836-44. DOI PubMed PMC 
55. Xing P, Zhang F, Wang G, et al. Incidence rates of immune-related adverse events and their correlation with response in advanced solid tumours treated with NIVO or NIVO+IPI: a systematic review and meta-analysis. J Immunother Cancer 2019;7:341. DOI PubMed PMC

56. Liu Y, Sethi NS, Hinoue T, et al. Comparative molecular analysis of gastrointestinal adenocarcinomas. Cancer Cell 2018;33:721735.e8. DOI PubMed PMC

57. Lauren P. The two histological main types of gastric carcinoma: diffuse and so-called intestinal-type carcinoma. An attempt at a histoclinical classification. Acta Pathol Microbiol Scand 1965;64:31-49. DOI PubMed

58. Kim ST, Cristescu R, Bass AJ, et al. Comprehensive molecular characterization of clinical responses to PD-1 inhibition in metastatic gastric cancer. Nat Med 2018;24:1449-58. DOI PubMed

59. Imamura $\mathrm{Y}$, Watanabe $\mathrm{M}$, Toihata $\mathrm{T}$, et al. Recent incidence trend of surgically resected esophagogastric junction adenocarcinoma and microsatellite instability status in Japanese patients. Digestion 2019;99:6-13. DOI PubMed

60. Imamura Y, Toihata T, Haraguchi I, et al. Immunogenic characteristics of microsatellite instability-low esophagogastric junction adenocarcinoma based on clinicopathological, molecular, immunological and survival analyses. Int J Cancer 2021;148:1260-75. DOI PubMed

61. Smyth EC, Wotherspoon A, Peckitt C, et al. Mismatch repair deficiency, microsatellite instability, and survival: an exploratory analysis of the medical research council adjuvant gastric infusional chemotherapy (MAGIC) trial. JAMA Oncol 2017;3:1197-203. DOI PubMed PMC

62. Lemery S, Keegan P, Pazdur R. First FDA approval agnostic of cancer site - when a biomarker defines the indication. N Engl J Med 2017;377:1409-12. DOI PubMed

63. Hansford S, Kaurah P, Li-Chang H, et al. Hereditary diffuse gastric cancer syndrome: CDH1 mutations and beyond. JAMA Oncol 2015;1:23-32. DOI PubMed

64. Fewings E, Larionov A, Redman J, et al. Germline pathogenic variants in PALB2 and other cancer-predisposing genes in families with hereditary diffuse gastric cancer without CDH1 mutation: a whole-exome sequencing study. Lancet Gastroenterol Hepatol 2018;3:489-98. DOI PubMed PMC

65. Sahasrabudhe R, Lott P, Bohorquez M, et al. Germline mutations in PALB2, BRCA1, and RAD51C, which regulate DNA recombination repair, in patients with gastric cancer. Gastroenterology 2017;152:983-6.e6. DOI PubMed PMC

66. Rokutan H, Hosoda F, Hama N, et al. Comprehensive mutation profiling of mucinous gastric carcinoma. J Pathol 2016;240:137-48. DOI PubMed

67. Dulak AM, Schumacher SE, van Lieshout J, et al. Gastrointestinal adenocarcinomas of the esophagus, stomach, and colon exhibit distinct patterns of genome instability and oncogenesis. Cancer Res 2012;72:4383-93. DOI PubMed PMC

68. Liu J, Lichtenberg T, Hoadley KA, et al. An integrated TCGA pan-cancer clinical data resource to drive high-quality survival outcome analytics. Cell 2018;173:400-16.e11. DOI PubMed PMC

69. Wong GS, Zhou J, Liu JB, et al. Targeting wild-type KRAS-amplified gastroesophageal cancer through combined MEK and SHP2 inhibition. Nat Med 2018;24:968-77. DOI PubMed PMC 